\title{
Review of: "A Distributed Ant Colony Optimization in Edge Detection"
}

\author{
Neelukumari Kumarasamy Subramaniam
}

Potential competing interests: The author(s) declared that no potential competing interests exist.

This work has compared MSE and PSR for ant colony algorithm and filtered ant colony optimization algorithms. But the result comparison with other algorithm as specified in related work has been failed to address. The author has failed to address this comparison. The mathematical model for filtered ant colony optimization algorithm is not addressed if it is provided it could be a better work. 